\title{
Dose-volume metrics associated with radiation pneumonitis after stereotactic body radiation therapy for lung cancer.
}

\section{$\operatorname{AUTHOR}(S):$}

Matsuo, Yukinori; Shibuya, Keiko; Nakamura, Mitsuhiro; Narabayashi, Masaru; Sakanaka, Katsuyuki; Ueki, Nami; Miyagi, Ken; ... Mizowaki, Takashi; Nagata, Yasushi; Hiraoka, Masahiro

\section{CITATION:}

Matsuo, Yukinori ...[et al]. Dose-volume metrics associated with radiation pneumonitis after stereotactic body radiation therapy for lung cancer.. International journal of radiation oncology, biology, physics 2012, 83(4): e545-e549

\section{ISSUE DATE:}

2012-07-15

\section{URL:}

http://hdl.handle.net/2433/158357

\section{RIGHT:}

(c) 2012 Elsevier Inc.; この論文は出版社版でありません。引用の際には 出版社版をご確認ご利用ください。; This is not the published version. Please cite only the published version. 
Title

Dose-volume metrics associated with radiation pneumonitis after stereotactic body radiation therapy for lung cancer

\section{Authors and affiliations}

Yukinori Matsuo, MD, PhD*; Keiko Shibuya, MD, PhD*; Mitsuhiro Nakamura, $\mathrm{PhD}^{*}$;

Masaru Narabayashi, MD*; Katsuyuki Sakanaka, MD*; Nami Ueki, MD*; Ken Miyagi,

MD*; Yoshiki Norihisa, MD*; Takashi Mizowaki, MD, PhD*; Yasushi Nagata, MD, PhD†;

Masahiro Hiraoka, MD, PhD*.

*Department of Radiation Oncology and Image-applied Therapy, Kyoto University, Kyoto, Japan.

$\dagger$ Division of Radiation Oncology, Hiroshima University Hospital, Hiroshima, Japan.

\section{Corresponding author}

Yukinori Matsuo, MD, PhD. Department of Radiation Oncology and Image-applied Therapy,

Graduate School of Medicine, Kyoto University, 54 Shogoin Kawahara-cho, Sakyo-ku,

Kyoto, 606-8507, Japan. 
Phone: +81-75-751-3762, fax: +81-75-771-9749

E-mail: ymatsuo@kuhp.kyoto-u.ac.jp

\section{Running title}

Dose-volume metrics and RP after SBRT for lung cancer

\section{Acknowledgments}

This research was supported by the Japan Society for the Promotion of Science (JSPS) through its "Funding Program for World-Leading Innovative R\&D on Science and Technology (FIRST Program)" initiated by the Council for Science and Technology Policy (CSTP), and by a Grant-in-Aid for Scientific Research (23791409) from the Ministry of

Education, Culture, Sports, Science, and Technology of Japan.

Part of this work was presented at the $52^{\text {nd }}$ Annual Meeting of the American Society for Radiation Oncology on October 31-November 4, 2010, at the San Diego Convention Center, San Diego, CA. 


\section{Conflict of Interest Notification:}

Yukinori Matsuo, none; Keiko Shibuya, none; Mitsuhiro Nakamura, none; Masaru

Narabayashi, none; Katsuyuki Sakanaka, none; Nami Ueki, none; Ken Miyagi, none; Yoshiki

Norihisa, none; Takashi Mizowaki, none; Yasushi Nagata, none; Masahiro Hiraoka, none. 
Title

\title{
Dose-volume metrics associated with radiation pneumonitis after stereotactic body radiation therapy for lung cancer
}

\begin{abstract}
Purpose: To identify dose-volume factors associated with radiation pneumonitis (RP) after stereotactic body radiotherapy (SBRT) for lung cancer.

Methods and Materials: This study analyzed 74 patients who underwent SBRT for primary lung cancer. The prescribed dose for SBRT was uniformly $48 \mathrm{~Gy}$ in four fractions at the isocenter. RP was graded according to the Common Terminology Criteria for Adverse Events (CTCAE) ver.3. Symptomatic RP was defined as Grade 2 or worse. Optimal cut-offs dividing the patient population into two subgroups based on the incidence of symptomatic RP were sought using the following dose-volume metrics: PTV volume (mL), mean lung dose (Gy), and V5, V10, V15, V20, V25, V30, V35, and V40 (\%) of both lungs excluding the PTV.
\end{abstract}

Results: With a median follow-up duration of 31.4 months, symptomatic RP was observed in 15 patients $(20.3 \%)$, including one patient with Grade 3. Optimal cut-offs for pulmonary 
dose-volume metrics were V25 and V20. These two factors were highly correlated with each other, and V25 was more significant. Symptomatic RP was observed in $14.8 \%$ of the patients with V25 $<4.2 \%$, and the rate was $46.2 \%$ in the remainder $(P=0.019)$. PTV volume was another significant factor. The symptomatic RP rate was significantly lower in the group with PTV $<37.7 \mathrm{~mL}$ compared with the larger PTV group $(11.1 \%$ vs. $34.5 \%, P=0.020)$. The patients into three subgroups- (1) PTV $<37.7 \mathrm{~mL}$; (2) PTV $\geq 37.7 \mathrm{~mL}$ and $\mathrm{V} 25<4.2 \%$; and (3) PTV $\geq 37.7 \mathrm{~mL}$ and V25 $\geq 4.2 \%$ - and the incidence of RP Grade 2 or worse was 11.1, 23.5, and 50.0\%, respectively $(P=0.013)$.

Conclusions: Lung V25 and PTV volume were significant factors associated with RP after SBRT.

\section{Keywords}

Radiation pneumonitis; stereotactic body radiation therapy; dose-volume analysis

\section{Running title}

Dose-volume metrics and RP after SBRT for lung cancer 


\section{Summary:}

To identify dose-volume factors associated with radiation pneumonitis (RP) after stereotactic body radiation therapy (SBRT) for lung cancer, this study analyzed 74 patients who underwent SBRT for primary lung cancer. RP Grade 2 or worse was observed in 15 patients (20.3\%). Lung V25 and PTV volume were significant factors. SBRT with PTV $\geq 37.7 \mathrm{~mL}$ and lung V25 $\geq 4.2 \%$ indicated a $50 \%$ risk of RP Grade 2 or worse. 


\section{Introduction}

Stereotactic body radiation therapy (SBRT) consists of two key features. One is its high accuracy, which allows delivery of a high dose to a very limited volume, while limiting the dose to normal tissues. The other key feature is hypofractionation. With conventional radiotherapy, schedules of 30-40 fractions are commonly used with a total dose of 60-74 Gy for lung cancer. SBRT is usually performed in a single fraction or 2-5 fractions with a much higher fractional dose (6-20 Gy). These key features of SBRT lead to high control rates of local tumors ${ }^{1,2}$. SBRT should improve the prognosis for inoperable or elderly patients with early stage non-small-cell lung cancer (NSCLC). ${ }^{3}$

The safety of SBRT is being confirmed in multi-institutional phase 2 trials for peripheral lung cancer in inoperable $e^{1,4}$ and operable patients ${ }^{2}$. In the Radiation Therapy Oncology Group (RTOG) trial 0236, ${ }^{1}$ protocol-specified treatment-related Grade 3 and 4 adverse events occurred in 12.7 and 3.6\%, respectively. No Grade 5 adverse events were reported. In the Nordic phase 2 study of SBRT, ${ }^{4}$ Grade 3 toxicity was seen in $21 \%$. No Grade 4 or 5 toxicity was reported. Nagata et al. reported Grade 3 toxicity in $6.2 \%$ of operable patients in the Japan Clinical Oncology Group (JCOG) $0403 .^{2}$ 
Radiation pneumonitis (RP) is one of the most common toxicities after SBRT, as well as after conventional radiotherapy to the lung. The reported rates of symptomatic RP after SBRT range from 9 to 28\%..$^{-11}$ Although most of the RP was Grade 2 and manageable, a few cases were severe, and there is a potential risk of mortality ${ }^{5}$. It is very important to develop a method to predict the risk of RP after SBRT for the lung.

The dose-volume metrics from a treatment plan might be a predictor for RP. In conventional radiotherapy for the lung, many dose-volume data are available, and dose constraints have been proposed to reduce the risk of pneumonitis. The Quantitative Analysis of Normal Tissue Effects in the Clinic (QUANTEC) recommended a lung V20 $\leq 30-35 \%$ and mean lung dose (MLD) $\leq 20-23$ Gy to limit the risk of RP to $\leq 20 \%$ in definitive radiotherapy for NSCLC with conventional fractionation. ${ }^{12}$ However, there is no established criterion regarding dose-volume factors in hypofractionated SBRT. Therefore, we searched for dose-volume factors associated with RP after SBRT for lung cancer.

\section{Materials and Methods}

This study analyzed consecutive 74 patients who underwent SBRT for pathology

proven NSCLC from September 2003 to March 2008. All patients were diagnosed as stage 
I lung cancer based on CT. The eligibility criteria for SBRT for stage I lung cancer were as follows: (1) surgery was contraindicated or refused; (2) maximal tumor diameter was 40 mm or less; (3) the tumor was not adjacent to mediastinal organs (spinal cord, esophagus, heart, and main bronchus); (4) the patient could remain stable for longer than 30 min with ECOG performance status (PS) of $0-2 ;$ (5) the patient had no active interstitial pneumonitis; and (6) written informed consent was obtained. The patient characteristics are shown in Table 1. For these patients, the prescribed dose of SBRT was uniformly 48 Gy in four fractions at the isocenter. A median in overall treatment time was 5 days (range, 4-12 days). Chemotherapy was not administered unless disease progression was confirmed.

\section{SBRT procedure}

Details of our SBRT procedure have been described in our previous reports. ${ }^{13,14}$ The patient's body was immobilized with a stereotactic body frame (Elekta AB, Stockholm, Sweden). Irradiation was performed with 6-MV x-ray beams from a linear accelerator (Clinac 2300 C/D; Varian Medical Systems, Palo Alto, CA) with multiple non-coplanar static ports (5-8 ports). The beams were shaped into a planning target volume (PTV) plus 5-mm margins using multi-leaf collimators. 
The SBRT was planned with the Eclipse (Varian Medical Systems) treatment planning system. An internal target volume (ITV) was determined using computed tomography (CT) with a slow-scan technique, considering tumor motion assessed by x-ray fluoroscopy. The PTV was defined as the ITV with a 5-mm margin for setup uncertainty. To delineate lung volumes, the segmentation wizard in Eclipse was applied to the slow-scan CT images with a threshold value of -300 Hounsfield units (HU). Then, the lung volumes were trimmed manually to remove overlapping regions involving the PTV and bronchi.

$R P$ grading

The patients were followed-up 1, 2, 4, 6, 9, and 12 months after the SBRT, then every 3 months for years 2 to 5 , and then every 6 months thereafter. CT was performed every 2-4 months in the first year, every 6 months between years 1 and 5 after the treatment, and annually thereafter.

Radiation pneumonitis was assessed according to the Common Terminology Criteria for Adverse Events (CTCAE) ver.3 for pneumonitis, which consists of the following grades: Grade 1, asymptomatic with radiographic findings only; Grade 2, symptomatic and not interfering with the activities of daily living (ADL); Grade 3, 
symptomatic, interfering with the ADL and $\mathrm{O}_{2}$ indicated; and Grade 4, life-threatening, ventilatory support indicated. We defined symptomatic RP as Grade 2 or worse.

\section{Evaluated data and statistical analysis}

The dose-volume metrics were re-calculated using the Analytical Anisotropic Algorithm (AAA) with the same monitor units as used in the clinical setting. The grid size for the calculation was $2.5 \times 2.5 \times 2.5 \mathrm{~mm}^{3}$.

The following dose-volume metrics were evaluated in this study: PTV volume $(\mathrm{mL})$, MLD (Gy), and V5, V10, V15, V20, V25, V30, V35, and V40, where Vd is the relative volume of normal lung (\%) that received more than a threshold dose of $d$ Gy. We defined normal lung as both lungs excluding the PTV.

The optimal cut-offs dividing the patient population into two subgroups based on the incidence of symptomatic RP were searched using the recursive partitioning method. After dividing the groups, the occurrence rates for symptomatic RP were compared using Fisher's exact test. R version 2.13.1 with the rpart package (R Foundation for Statistical Computing, Vienna, Austria) was used for statistical analyses. Statistical significance was defined as $P<0.05$. 


\section{Results}

With a median follow-up duration of 31.4 (range 4.2-65.0) months, symptomatic RP was observed in 15 patients (20.3\%), consisting of 14 patients with Grade 2 and one with Grade 3. RP was diagnosed based on symptoms with radiologic findings. A median time to symptomatic RP was 4.5 months (range, 1.1-16.1 months).

Table 2 summarizes the dose-volume metrics of the PTV and normal lung. The PTV ranged from 9.9 to $86.6 \mathrm{~mL}$ (median $32.5 \mathrm{~mL}$ ). The median MLD was 3.2 (range 1.6-7.6) Gy. The median lung V20 and V25 were $4.4 \%$ and $2.9 \%$, respectively. The lung metrics (MLD and V5-40) were highly correlated with each other, with correlation coefficients $(R)$ of 0.61-0.99. In contrast, the correlations between the PTV volume and lung metrics were weak $(R=0.37-0.59)$. The PTV volume, MLD, V20 and V25 were respectively $42.1 \mathrm{~mL}$, 3.7 Gy, 5.4\% and 3.8\% in mean for Grade 2 RP patients. Those values were respectively $38.1 \mathrm{~mL}, 4.0 \mathrm{~Gy}, 5.0 \%$ and $3.3 \%$ for a Grade 3 patient.

The optimal cut-offs for the dose-volume metrics are shown in Table 3. Lung V25 and V20 were significant factors, and V25 was more significant. The rate of symptomatic RP was $14.8 \%$ in the patients with V25<4.2\%, whereas the rate was $46.2 \%$ in the remainder 
$(P=0.019)$. The PTV volume was another significant factor. The symptomatic RP rate was significantly lower in the group with PTV $<37.7 \mathrm{~mL}$ compared with the larger PTV group (11.1\% vs. $34.5 \%, P=0.020)$. On classifying the patient population into three subgroups(1) PTV <37.7 mL $(n=45)$; (2) PTV $\geq 37.7 \mathrm{~mL}$ and V25 <4.2\% $(n=17)$; and (3) PTV $\geq 37.7$ $\mathrm{mL}$ and $\mathrm{V} 25 \geq 4.2 \%(n=12)$ - the incidence of symptomatic RP was $11.1,23.5$, and $50.0 \%$, respectively $(P=0.013)$.

\section{Discussion}

We investigated the dosimetric factors associated with symptomatic RP ( $\geq$ Grade 2 ) after SBRT, and found that PTV and lung V25 were significant factors. The optimal cut-offs for these factors (PTV $<37.7 \mathrm{~mL}$ and V25 <4.2\%) were much lower than prespecified constraints which other SBRT trials used to mainly limit the risk of Grade 3 toxicities (e.g. MLD <18Gy, lung V40 <100mL, V15<25\% and V20<20\% for the JCOG 0403 trial; and lung V20<10\% for the RTOG 0236). As only one patient suffered from Grade 3 RP in our cohort, it was difficult to build a model for predicting severe RP (e.g., Grade 3 or worse). Severe RP is uncommon after SBRT for the lung. Grade 3 RP was observed in $3.6 \%$ of the patients in the RTOG $0236^{1}$ and in $3.1 \%$ of the operable patients in 
JCOG 0403. ${ }^{2}$ Baumann et al. reported that no one developed Grade 3 pneumonitis in their phase 2 trial of SBRT. ${ }^{4}$

Table 4 summarizes reports that focused on the dose-volume metrics associated with RP Grade 2 or worse after SBRT. The RP rates varied from 9.4-28.0\%, and the suggested dose-volume factors for RP differed among the reports. This variation might be caused by differences in the PTV volume, dose-fractionation schedule, and RP scoring system.

The scoring criteria for RP differ among toxicity-grading systems. Steroid use for pneumonitis is scored as Grade 3 in the RTOG system, whereas the Common Toxicity Criteria (CTC) ver.2 scores symptomatic patients requiring steroids as Grade 2. CTCAE ver.3 removed steroid use from the pneumonitis scoring system. From CTCAE ver.3 to ver.4, the pneumonitis score was modified slightly. Grade 2 pneumonitis in CTCAE ver.4 was defined as "symptomatic, medical intervention indicated or limiting instrumental ADL." The number of patients considered to have RP depends on which system is used to evaluate RP. Tucker et al. retrospectively evaluated 442 patients who received definitive radiotherapy for NSCLC using the three RP grading systems: RP Grade 2 or worse was observed in 129 (29\%), 109 (25\%), and 195 (44\%) patients according to RTOG, CTC ver.2 and CTCAE ver.3, respectively. ${ }^{15}$ The rate of Grade 2-3 RP according to CTCAE ver.3 
seemed to be slightly higher $(20.3 \%)$ in our study compared with other studies that evaluated it using CTC ver.2 or the RTOG system. Nevertheless, we administered steroids to six patients $(8.1 \%)$ only. Scoring systems should be considered when interpreting the results regarding $\mathrm{RP}$.

This study indicated that a large PTV is a significant risk factor for symptomatic RP after SBRT. Ong et al. treated large tumors in 18 patients with a PTV >80 mL (median 137 mL) with SBRT using volumetric modulated arc therapy. ${ }^{9}$ They reported five patients (27.8\%) with RP Grade 2-3. We speculate that the large PTV volume was one of the reasons for their relatively high risk of pneumonitis. From our results, minimizing the PTV is one way to limit the RP risk. When a lung tumor has large respiratory motion, respiration management will contribute to the prevention of RP by reducing the PTV.

To correct for differences in dose-fractionation, three reports ${ }^{6-8}$ in Table 4 used normalized total doses (NTD), which were equivalent doses in 2-Gy fractions in terms of pulmonary toxicity. Borst et al. evaluated the relationship between the MLD and incidence of RP after SBRT. ${ }^{7}$ They calculated the MLD in the NTD form using the linear-quadratic model with an $\alpha / \beta$ ratio of 3 Gy. A significant dose-response relationship was found between RP and MLD. We used a uniform dose fractionation of $48 \mathrm{~Gy}$ in four fractions for 
all of our patients, and we evaluated dosimetric factors in the form of a nominal dose. Although we cannot apply normalization to our data exactly, the median MLD was roughly estimated to be 3.7 Gy in NTD. The usefulness of NTD evaluation for pulmonary toxicity after SBRT should be validated in future studies.

Heterogeneity correction and the definition of lung volume may influence the dose-volume factors of the lung. De Jaeger et al. evaluated differences between the equivalent-pathlength (EPL) and convolution-superposition (CS) algorithms in MLD and V20 for 68 patients treated with conformal radiotherapy for NSCLC. ${ }^{16}$ MLD and V20 differed between the two algorithms by $16.9 \%$ and $12.0 \%$ on average, respectively. The $\mathrm{TD}_{50}$ for RP Grade 2 or worse in MLD was estimated to be 34.1 Gy with the EPL algorithm, whereas the value was 29.2 Gy with the CS algorithm. Because most related reports have used convolution-superposition algorithms, we applied the AAA, which is a convolution-superposition algorithm, to recalculate the dose-volume data so as to limit the influences of heterogeneity correction.

Our definition of lung volume was both lungs segmented with a threshold value of -300 HU on slow-scan CT from which PTV was excluded. Lung volume can vary depending on which lung is evaluated (ipsilateral, contralateral, or bilateral), exclusion of 
tumor volume (GTV or PTV), type of planning CT (slow-scan CT, 4D CT, or breath-hold CT), and the threshold HU for segmentation. When the PTV is subtracted from the lung volume, a high-dose volume within the PTV margin is not evaluated as dose-volume factors for the lung. These conditions were not always stated in the previous papers, and future articles should include this information.

Factors other than the dose-volume metrics also affect the occurrence of pneumonitis after SBRT. The serum level of Krebs von den Lungen-6 (KL-6) is a predictor of RP. Hara et al. evaluated 16 patients who received single-fraction SBRT with 20-35 Gy. ${ }^{17}$ The relative increase in the serum KL- 6 between before and at 2 months after SBRT was significantly correlated with the occurrence of Grade 3 RP by the RTOG criteria. Iwata et al. reported that the pretreatment serum KL-6 levels, gender, and PTV volume were correlated with symptomatic RP in a univariate analysis, and the pretreatment KL-6 levels remained significant in a multivariate analysis. ${ }^{18}$ They concluded that patients with a pretreatment KL-6 level $\geq 300 \mathrm{U} / \mathrm{mL}$ should be followed carefully for the occurrence of RP. CT or X-ray imaging before and after SBRT should help to predict severe RP. Yamashita et al. recommended prescreening of interstitial pneumonitis on $\mathrm{CT}$, in addition to checking the serum KL-6 and surfactant protein-D (SP-D) levels to limit the risks of severe RP. ${ }^{19}$ They 
reported that after introducing the prescreening, the occurrence rate of RP Grade 4-5 decreased from $18.8 \%$ to $3.5 \%$. Takeda et al. reported that the early appearance of RP on chest X-ray after SBRT was correlated with the severity of $\mathrm{RP}^{20}$ The radiographic appearance of RP during the initial 2 months indicated a $40 \%$ risk for Grade 3 RP, whereas the risk was only $1.2 \%$ when the radiologic change appeared 3 months after SBRT. Considering biomarkers (KL-6 and SP-D) and radiologic imaging as well as dose-volume factors helps us to limit the risks for severe pneumonitis after SBRT.

Our study has some limitations. First, this study was based on a retrospective review; thus, it was prone to selection bias. Second, the number of RP events was limited in the present study. Prospective data are awaited to investigate a correlation between pulmonary toxicities and dosimetric factors. The other limitation was that clinical factors other than dose-volume metrics were not taken into consideration, as described above.

In conclusion, this study found that the lung V25 and PTV volumes were significant factors associated with RP after SBRT. SBRT with PTV $\geq 37.7 \mathrm{~mL}$ and lung V25 $\geq 4.2 \%$ indicated a $50 \%$ risk of RP Grade 2 or worse. 


\section{References}

1. Timmerman R, Paulus R, Galvin J, et al. Stereotactic body radiation therapy for inoperable early stage lung cancer. JAMA. 2010;303:1070-6.

2. Nagata Y, Hiraoka M, Shibata T, et al. A Phase II Trial of Stereotactic Body Radiation Therapy for Operable T1N0M0 Non-small Cell Lung Cancer: Japan Clinical Oncology Group (JCOG0403). Int J Radiat Oncol Biol Phys. 2010;78:S27-S28.

3. Palma D, Visser O, Lagerwaard FJ, et al. Impact of introducing stereotactic lung radiotherapy for elderly patients with stage I non-small-cell lung cancer: a population-based time-trend analysis. J Clin Oncol. 2010;28:5153-9.

4. Baumann P, Nyman J, Hoyer M, et al. Stereotactic body radiotherapy for medically inoperable patients with stage I non-small cell lung cancer - a first report of toxicity related to $\mathrm{COPD/CVD}$ in a non-randomized prospective phase II study. Radiother Oncol.

2008;88:359-67. 
5. Yamashita H, Nakagawa K, Nakamura N, et al. Exceptionally high incidence of symptomatic grade 2-5 radiation pneumonitis after stereotactic radiation therapy for lung tumors. Radiat Oncol. 2007;2:21.

6. Ricardi U, Filippi AR, Guarneri A, et al. Dosimetric predictors of radiation-induced lung injury in stereotactic body radiation therapy. Acta Oncol. 2009;48:571-7.

7. Borst GR, Ishikawa M, Nijkamp J, et al. Radiation pneumonitis in patients treated for malignant pulmonary lesions with hypofractionated radiation therapy. Radiother Oncol. 2009;91:307-13.

8. Guckenberger M, Baier K, Polat B, et al. Dose-response relationship for radiation-induced pneumonitis after pulmonary stereotactic body radiotherapy. Radiother Oncol. 2010;97:65-70.

9. Ong CL, Palma D, Verbakel WFAR, Slotman BJ, Senan S. Treatment of large stage I-II lung tumors using stereotactic body radiotherapy (SBRT): planning considerations and early toxicity. Radiother Oncol. 2010;97:431-6. 
10. Barriger RB, Forquer J a, Brabham JG, et al. A dose-volume analysis of radiation pneumonitis in non-small cell lung cancer patients treated with stereotactic body radiation therapy. Int J Radiat Oncol Biol Phys. 2012;82:457-62.

11. Stauder MC, Macdonald OK, Olivier KR, et al. Early pulmonary toxicity following lung stereotactic body radiation therapy delivered in consecutive daily fractions. Radiother Oncol. 2011;99:166-71.

12. Marks LB, Bentzen SM, Deasy JO, et al. Radiation dose-volume effects in the lung. Int J Radiat Oncol Biol Phys. 2010;76:S70-6.

13. Takayama K, Nagata Y, Negoro Y, et al. Treatment planning of stereotactic radiotherapy for solitary lung tumor. Int J Radiat Oncol Biol Phys. 2005;61:1565-71.

14. Hiraoka M, Matsuo Y, Takayama K. Stereotactic body radiation therapy for lung cancer: achievements and perspectives. Jpn J Clin Oncol. 2010;40:846-54.

15. Tucker SL, Jin H, Wei X, et al. Impact of toxicity grade and scoring system on the relationship between mean lung dose and risk of radiation pneumonitis in a large cohort of patients with non-small cell lung cancer. Int J Radiat Oncol Biol Phys. 2010;77:691-8. 
16. De Jaeger K, Hoogeman MS, Engelsman M, et al. Incorporating an improved dose-calculation algorithm in conformal radiotherapy of lung cancer: re-evaluation of dose in normal lung tissue. Radiother Oncol. 2003;69:1-10.

17. Hara R, Itami J, Komiyama T, Katoh D, Kondo T. Serum levels of KL-6 for predicting the occurrence of radiation pneumonitis after stereotactic radiotherapy for lung tumors.

Chest. 2004;125:340-4.

18. Iwata H, Shibamoto Y, Baba F, et al. Correlation between the serum KL-6 level and the grade of radiation pneumonitis after stereotactic body radiotherapy for stage I lung cancer or small lung metastasis. Radiother Oncol. 2011;101:267-70.

19. Yamashita H, Kobayashi-Shibata S, Terahara A, et al. Prescreening based on the presence of CT-scan abnormalities and biomarkers (KL-6 and SP-D) may reduce severe radiation pneumonitis after stereotactic radiotherapy. Radiat Oncol. 2010;5:32.

20. Takeda A, Ohashi T, Kunieda E, et al. Early graphical appearance of radiation pneumonitis correlates with the severity of radiation pneumonitis after stereotactic body 
Matsuo Y, et al. Dose-volume metrics and RP after SBRT for lung cancer

radiotherapy (SBRT) in patients with lung tumors. Int J Radiat Oncol Biol Phys.

2010;77:685-90. 
Tables

\begin{tabular}{ll}
\hline Sex & \\
Male & 55 \\
Female & 19 \\
\hline Age (y) & $77(63-88)$ \\
$\quad$ Median (range) & \\
\hline ECOG performance status & 37 \\
0 & 30 \\
1 & 7 \\
2 & \\
\hline Operability & 24 \\
Operable & 50 \\
Inoperable & \\
\hline Histology & 36 \\
Adenocarcinoma & 30 \\
Squamous cell carcinoma & 8 \\
Others* & \\
\hline T-stage (the 7th UICC staging) & 26 \\
T1a & 27 \\
T1b & 21 \\
T2a & \\
\hline
\end{tabular}

Table 1. Patient characteristics

* Others included 3 large cell carcinomas and 5 non-small cell carcinomas not other specified. 


\begin{tabular}{llc}
\hline & & Median (range) \\
\hline PTV & Volume (mL) & $32.5(9.9-86.6)$ \\
\hline Lung & Mean dose (Gy) & $3.2(1.6-7.6)$ \\
& V40 (\%) & $0.7(0-2.9)$ \\
& V35 (\%) & $1.4(0.2-4.8)$ \\
& V30 (\%) & $2.0(0.5-6.6)$ \\
& V25 $(\%)$ & $2.9(0.9-9.5)$ \\
& V20 (\%) & $4.4(1.7-13.1)$ \\
& V15 (\%) & $6.7(2.6-18.2)$ \\
& V10 (\%) & $10.3(4.4-25.1)$ \\
V5 $(\%)$ & $16.7(7.1-37.3)$ \\
\hline
\end{tabular}

Table 2. Median and range for the dose-volume metrics 


\begin{tabular}{lcccc}
\hline & Cut-off & \multicolumn{2}{c}{ Symptomatic RP } & \multirow{2}{*}{ P-values } \\
\cline { 3 - 4 } & values & <cut-off & $\geq$ cut-off & \\
\hline PTV (mL) & 37.7 & $11.1 \%$ & $34.5 \%$ & $0.020 *$ \\
MLD (Gy) & 4.7 & $17.2 \%$ & $40.0 \%$ & 0.110 \\
V40 (\%) & 1.6 & $17.6 \%$ & $50.0 \%$ & 0.093 \\
V35 (\%) & 1.9 & $15.3 \%$ & $40.0 \%$ & 0.066 \\
V30 (\%) & 2.8 & $15.3 \%$ & $40.0 \%$ & 0.066 \\
V25 (\%) & 4.2 & $14.8 \%$ & $46.2 \%$ & $0.019 *$ \\
V20 (\%) & 5.8 & $15.0 \%$ & $42.9 \%$ & $0.030 *$ \\
V15 (\%) & 4.9 & $5.3 \%$ & $25.5 \%$ & 0.096 \\
V10 (\%) & 10.1 & $11.4 \%$ & $28.2 \%$ & 0.089 \\
V5 (\%) & 26.8 & $17.9 \%$ & $42.9 \%$ & 0.143 \\
\hline
\end{tabular}

Table 3. Optimal cut-off values and crude rates of symptomatic RP Abbreviations: $\mathrm{RP}=$ radiation pneumonitis, $\mathrm{MLD}=$ mean lung dose Asterisks (*) mean statistical significance. 


\begin{tabular}{|c|c|c|c|c|c|c|c|c|}
\hline Authors & Year & $\begin{array}{l}\text { No. of } \\
\text { patients }\end{array}$ & $\begin{array}{l}\text { Dose fractionation } \\
\text { [total dose/ } \\
\text { fractions] }\end{array}$ & $\begin{array}{l}\text { Heterogeneity } \\
\text { correction } \\
\text { algorithm }\end{array}$ & $\begin{array}{l}\text { PTV volume }(\mathrm{mL}) \\
\text { [median (range)] }\end{array}$ & $\begin{array}{l}\text { RP Gr.2 or } \\
\text { worse } \\
\text { [crude rate] }\end{array}$ & $\begin{array}{l}\text { Scoring } \\
\text { system }\end{array}$ & $\begin{array}{l}\text { Suggested } \\
\text { factors }\end{array}$ \\
\hline Yamashita $^{5}$ & 2007 & 25 & $48 \mathrm{~Gy} / 4-6 \mathrm{fr}$ & $\mathrm{CC}$ & $43.9(7.5-239.4)$ & $28.0 \%$ & CTCAE v3 & $\mathrm{CI}$ \\
\hline Ricardi ${ }^{6}$ & 2009 & 60 & $\begin{array}{l}45 \mathrm{~Gy} / 3 \mathrm{fr} \\
\text { or } 26 \mathrm{~Gy} / 1 \mathrm{fr}\end{array}$ & $\mathrm{CC}$ & $N A$ & $14.3 \%$ & RTOG & $\begin{array}{l}\text { MLD† (ips. } \\
\text { lung-CTV) }\end{array}$ \\
\hline Borst $^{7}$ & 2009 & 128 & $35-60$ Gy/ 4-8 fr & $\mathrm{CS}$ & $\begin{array}{l}9.6(0.2-106.9) \\
\text { in GTV }\end{array}$ & $10.9 \%$ & CTC v2 & $\begin{array}{l}\text { MLD† (bil. } \\
\text { lung-GTV) }\end{array}$ \\
\hline Guckenberger & 2010 & 59 & $\begin{array}{c}26 \mathrm{~Gy} / 1 \mathrm{fr} \\
\text { or } 37.5 \mathrm{~Gy} / 3 \mathrm{fr}\end{array}$ & AAA & $33(2-236)$ & $18.6 \%$ & SWOG & $\begin{array}{c}\text { MLD } \dagger \text { and } \\
\text { V2.5-50 (ips. } \\
\text { lung-CTV) }\end{array}$ \\
\hline $\mathrm{Ong}^{9}$ & 2010 & 18 & $\begin{array}{l}55 \mathrm{~Gy} / 5 \mathrm{fr} \\
\text { or } 60 \mathrm{~Gy} / 8 \mathrm{fr}\end{array}$ & AAA & $137(87-286)$ & $27.8 \%$ & CTCAE v4 & $\begin{array}{l}\text { V5 (con. } \\
\text { lung) }\end{array}$ \\
\hline Barriger $^{10}$ & 2010 & 251 & $24-66 \mathrm{~Gy} / 3-5 \mathrm{fr}$ & $N A^{*}$ & $48.3(8-401)$ & $9.4 \%$ & CTC v2 & $\begin{array}{l}\text { MLD and } \\
\text { V20 (bil. } \\
\text { lung-GTV) }\end{array}$ \\
\hline Stauder $^{11}$ & 2011 & 84 & $32-60 \mathrm{~Gy} / 3-5 \mathrm{fr}$ & $N A^{*}$ & $42.9(5.3-321.5)$ & $12.5 \%$ & CTCAE v3 & $\begin{array}{l}\text { PTV } \\
\text { maximal } \\
\text { dose }\end{array}$ \\
\hline Present study & & 74 & $48 \mathrm{~Gy} / 4 \mathrm{fr}$ & AAA & $32.5(9.9-86.6)$ & $20.3 \%$ & CTCAE v3 & $\begin{array}{l}\text { PTV volume } \\
\text { and V25 (bil. } \\
\text { lung-PTV) }\end{array}$ \\
\hline
\end{tabular}


Table 4. Summary of reports on dose-volume factors associated with radiation pneumonitis Grade 2 or worse after stereotactic body radiation therapy

* The authors reported that tissue heterogeneity was corrected in some of the patients, but information on the heterogeneity correction algorithm was not available.

$\dagger$ The MLD was evaluated using the normalized total dose, which is the equivalent dose in 2-Gy fractions.

Abbreviations: $\mathrm{RP}=$ radiation pneumonitis, $\mathrm{NA}=$ not available, $\mathrm{CC}=$ collapsed cone convolution superposition, $\mathrm{CS}=$ convolution superposition, $\mathrm{AAA}=$ analytical anisotropic algorithm, $\mathrm{CTC}=$ common toxicity criteria, $\mathrm{CTCAE}=$ common terminology criteria for adverse events, RTOG = Radiation Therapy Oncology Group, SWOG = Southwest Oncology Group, CI = conformity index, MLD = mean lung dose, ips. $=$ ipsilateral, con. $=$ contralateral, bil. $=$ bilateral 